\title{
An Overview of High-Entropy Alloys as Biomaterials
}

\author{
Diogo Castro ${ }^{1}$, Pedro Jaeger ${ }^{2} \mathbb{D}$, Ana Catarina Baptista ${ }^{1}\left(\mathbb{D}\right.$ and João Pedro Oliveira ${ }^{3, * \mathbb{C}}$ \\ 1 CENIMAT/I3N, Departamento de Ciência dos Materiais, Faculdade de Ciências e Tecnologia, FCT, \\ Universidade Nova de Lisboa, 2829-516 Campus de Caparica, Portugal; ddp.castro@campus.fct.unl.pt (D.C.); \\ anacbaptista@fct.unl.pt (A.C.B.) \\ 2 Instituto de Soldagem e Mecatrônica LABSOLDA, Universidade Federal de Santa Catarina-UFSC, \\ Florianópolis 88040-900, Brazil; pedro.jaeger@posgrad.ufsc.br \\ 3 UNIDEMI, Department of Mechanical and Industrial Engineering, NOVA School of Science and Technology, \\ Universidade NOVA de Lisboa, 2829-516 Caparica, Portugal \\ * Correspondence: jp.oliveira@fct.unl.pt
}

Citation: Castro, D.; Jaeger, P.;

Baptista, A.C.; Oliveira, J.P. An Overview of High-Entropy Alloys as Biomaterials. Metals 2021, 11, 648. https://doi.org/10.3390/met11040648

Academic Editors: Weidong Li and Leszek Adam Dobrzanski

Received: 3 March 2021

Accepted: 31 March 2021

Published: 15 April 2021

Publisher's Note: MDPI stays neutral with regard to jurisdictional claims in published maps and institutional affiliations.

Copyright: (c) 2021 by the authors. Licensee MDPI, Basel, Switzerland. This article is an open access article distributed under the terms and conditions of the Creative Commons Attribution (CC BY) license (https:/ / creativecommons.org/licenses/by/ $4.0 /)$.

\begin{abstract}
High-entropy alloys (HEAs) have been around since 2004. The breakthroughs in this field led to several potential applications of these alloys as refractory, structural, functional, and biomedical materials. In this work, a short overview on the concept of high-entropy alloys is provided, as well as the theoretical design approach. The special focus of this review concerns one novel class of these alloys: biomedical high-entropy alloys. Here, a literature review on the potential high-entropy alloys for biomedical applications is presented. The characteristics that are required for these alloys to be used in biomedical-oriented applications, namely their mechanical and biocompatibility properties, are discussed and compared to commercially available Ti6Al4V. Different processing routes are also discussed.
\end{abstract}

Keywords: high-entropy alloys; biomedical materials

\section{Introduction}

High-entropy alloys are loosely defined as alloys containing at least five principal elements, each having an atomic percentage ranging between 5 and $35 \%$, according to Yeh et al. [1], who alongside Cantor et al. [2], first discovered these alloys. There are other proposed parameters and definitions, such as the non-existence of intermetallic phases on a solid solution that would make an high-entropy alloy [3]. For these materials, high entropy refers to the high value of mixing entropy between equimolar metal alloys, conventionally, more than $1.5 \mathrm{R}$, resulting in a high probability of forming random solid solutions during solidification instead of forming intermetallic compounds or segregated states. Similarly, a medium-entropy alloy [4] is defined as a material composed of two to four principal elements or having a mixing entropy between $\mathrm{R}$ and $1.5 \mathrm{R}$ [5].

The origin of studies related to high-entropy alloys can be traced back to the 1980s though the first published works with actual alloys only came out in 2004, as previously described [1,2]. These pioneer works marked the beginning of an increasing number of studies regarding this new branch of alloy development [6]. Among some of the key properties exhibited by these alloys in their early years, one can account for their excellent mechanical behavior, the high corrosion resistance, and the suggestion of the existence of effects such as the high-entropy effect, lattice distortion effect, and slow diffusion effect [1].

Ranganathan [7] described the limitations on the development of multicomponent alloys, since the amount of information needed to study these alloys as the number of components increase was difficult to manage, and because by adding processing variables, it would be of astronomical proportions. Although this turned out to be true, in recent years, there has been an increase in the number of ways currently available for the fast and reliable identification of new alloys compositions of interest. 
High-entropy alloys are also known to possess unique microstructural features giving rise to improved properties over conventional engineering alloys. There are several contributions to the extraordinary properties of high-entropy alloys. These contributions are known as the four core effects, as described by Yeh [8].

Figure 1 represents the relationships between composition, structure and microstructure, and physical and mechanical properties of high-entropy alloys, providing the context in which the four core effects take place [6,8-11].

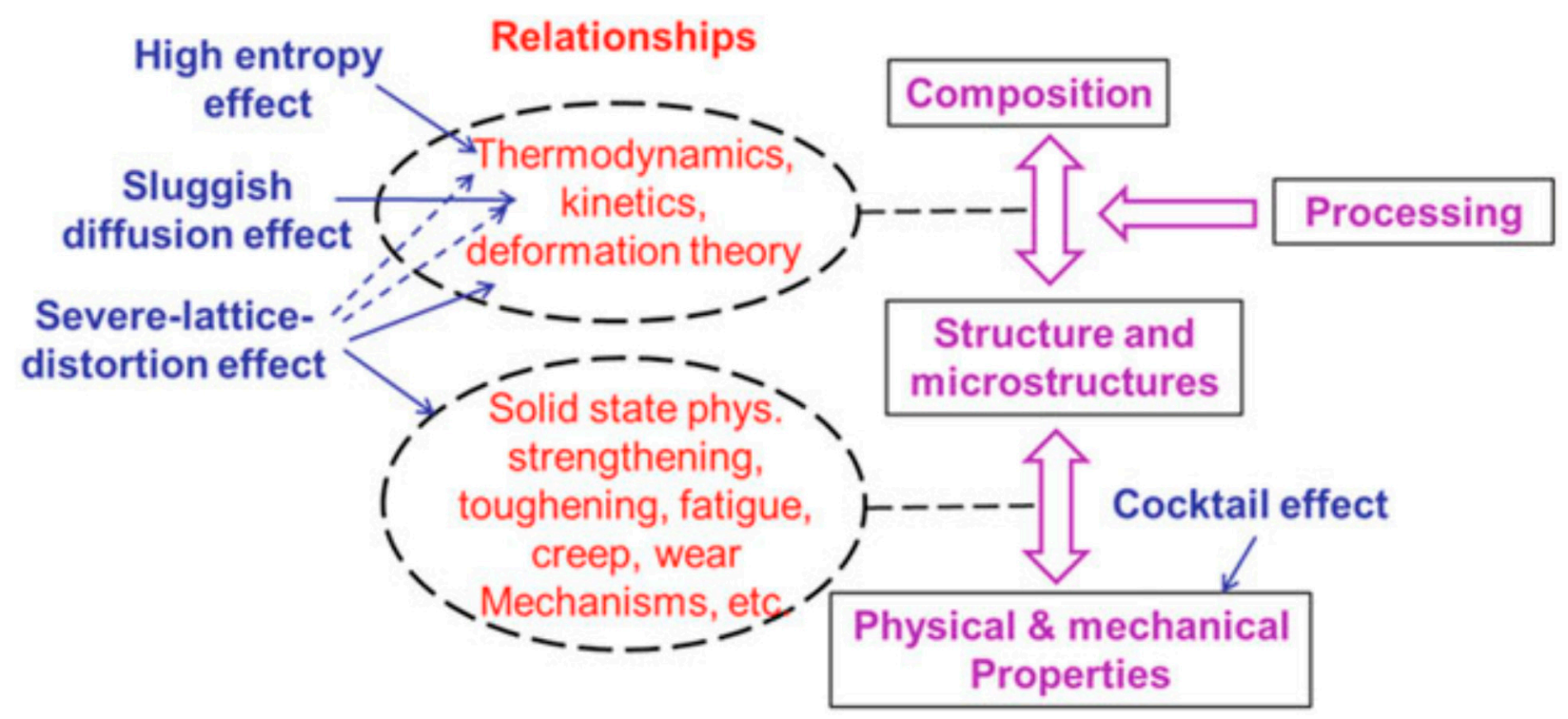

Figure 1. The scheme of physical metallurgy showing areas that are influenced by the four core effects exhibited by highentropy alloys (from [6]). Reprinted with permission from Springer Nature: eBook "High-Entropy Alloys-Fundamentals and Applications", Copyright 2021, License: 5021540456140.

The four core effects are as follows: high-entropy effect; sluggish diffusion; severe lattice distortion; and cocktail effect. The high-entropy effect is where, contrary to what was previously thought to be the case, mixing equimolar chemically compatible elements narrows down the possible solid solution phases of an alloy. The high mixing entropy in multiprincipal elements lowers the Gibbs free energy for random and partially ordered solid solutions to compete with intermetallic compounds on attaining equilibrium states at high temperatures. On the other hand, having high-entropy alloys with a more significant difference in the enthalpy of mixing between unlike atom pairs, leads to the formation of more than two phases [12]. The atomic size difference parameter $(\delta)$ also plays a role in this and is compared alongside the entropy of mixing $\left(\Delta S_{\text {mix }}\right)$ and enthalpy of mixing $\left(\Delta H_{\text {mix }}\right)$ in describing trends, setting phase selection rules [13], and describing the order-disorder competition in these alloys. The severe lattice distortion effect, where different atomic sizes, bonding energies, and crystal structure tendencies among the constituent elements all contribute to the lattice distortion, typically results in a large solution hardening effect. This effect leads to enhanced properties such as strength and hardness, and the temperature dependance of these properties is also consequently low. The sluggish diffusion effect, where the number of principal elements in the system's matrix is proportional to the degree of sluggish diffusion, leads to higher recrystallization temperatures and creep resistances, slower grain growth and particle coarsening, and ease of getting supersaturated states and fine precipitates. Finally, the cocktail effect is where each phase, being a multiprincipal element solid solution, is regarded as both an atomic-scale and microscale composite, having multiprincipal element composite effects and multiphase composite effects, respectively. Due to the interactions between the different elements and the severe lattice distortion, the rule of mixtures of the different elements underpredicts the alloys' changes by a substantial amount. 


\subsection{Alloy Design Strategy: The Theoretical Approach}

Parameters such as enthalpy of mixing, entropy of mixing, melting points, atomic size difference, and valence electron concentration are used as phase formation rules for highentropy alloys. These rules and parameters allow researchers to predesign a high-entropy alloy with the characteristics that best suit their envisaged application.

\subsubsection{Enthalpy and Entropy of Mixing}

To understand these two concepts, a couple of assumptions are required to be established. When equal atom size and loose atomic packing are assumed, the configuration entropy of mixing of an alloy is equivalent to that of an ideal gas. Moreover, there are competing states in the solid state of an alloy, which are elemental phases, intermetallic compounds, and solid solution phases (random solid solutions and partially ordered solid solutions). The one with the lowest free energy at a given temperature and pressure will be predominant in the equilibrium state, and so, if kinetic factors are not involved, phase formation is thermodynamically controlled by the Gibbs free energy, $G$ :

$$
\Delta G_{m i x}=\Delta H_{m i x}-T \times \Delta S_{m i x}
$$

Therefore, the configurational entropy of mixing, under said assumptions, is defined as follows:

$$
\Delta S_{\text {mix }}=-R \sum_{i=1}^{n} x_{i} \times \ln \left(x_{i}\right)
$$

with $R$ being the gas constant and $x_{i}$ the molar fraction of the $i$ th element, and the enthalpy of mixing for multicomponent alloys [13] is defined as follows:

$$
\Delta H_{m i x}=\sum_{i=1, i \neq j}^{n} 4 \Delta H_{A B}^{m i x} x_{i} x_{j}
$$

where $\Delta H_{A B}^{m i x}$ is the enthalpy of mixing for a binary equiatomic $A B$ alloy and $x_{i}$ and $x_{j}$ are the molar fractions of the $i$ th element.

The enthalpy of mixing is proportional to the number of bonds of unlike atoms, and completely ordered structures have a higher number of unlike atom pair bonds than that of random solid solutions, and although this difference lowers with the increase in the number of principal elements in an equiatomic high-entropy alloy, the number of unlike atom pair bonds in random solid solutions will never be the same or higher than that of intermetallic compounds. The reason a solid solution manages to compete with intermetallic compounds is because the entropy of mixing for completely ordered structures is $\approx 0$, while in disordered structures such as random solid solutions, the $\Delta S_{\text {mix }}$ equals $R \ln n$ [10].

Of course, equal atom size and loose atomic packing cannot be assumed for real alloys, and so the above definition has its limits. For this, another parameter is used, considering atomic composition, the atom size, and the overall packing density. According to Mansoori et al. [14], the total configurational entropy of mixing $S_{T}$ per sphere for a hard sphere system is defined as follows:

$$
S_{T}\left(c_{i}, r_{i}, \xi\right)=S_{C}\left(c_{i}\right)+S_{E}\left(c_{i}, r_{i}, \xi\right)
$$

where $S_{C}=-k_{B} \sum_{i=1}^{n} c_{i} \ln c_{i}$ is the equivalent to $\Delta S_{m i x}$, the configurational entropy of mixing for an ideal solution. $S_{E}$ is a function of the atomic composition, $c_{i}$, the atom size $r_{i}$, and the overall packing density, $\xi$, and is defined as the excessive entropy of mixing [14-16]. Thus, for high values of $\xi$, non-equiatomic compositions, and different atomic sizes between constituent elements, there is a correction provided by $S_{E}$ that makes $S_{T}$ a more precise value of configurational entropy of mixing, whereas for high temperatures, $\xi$ is negligible enough so that $\Delta S_{\text {mix }}$ remains a good approximation. The variation of the total entropy 
of mixing, $S_{T}$, with the atomic packing fraction, $\xi$, for different high-entropy alloys is detailed in Figure 2. It is worth noting that with $S_{T}$, the difference in the total entropy of mixing for different high-entropy alloy systems with the same number of principal elements, becomes increasingly evident when atomic packing fraction gets higher with decreasing temperature.

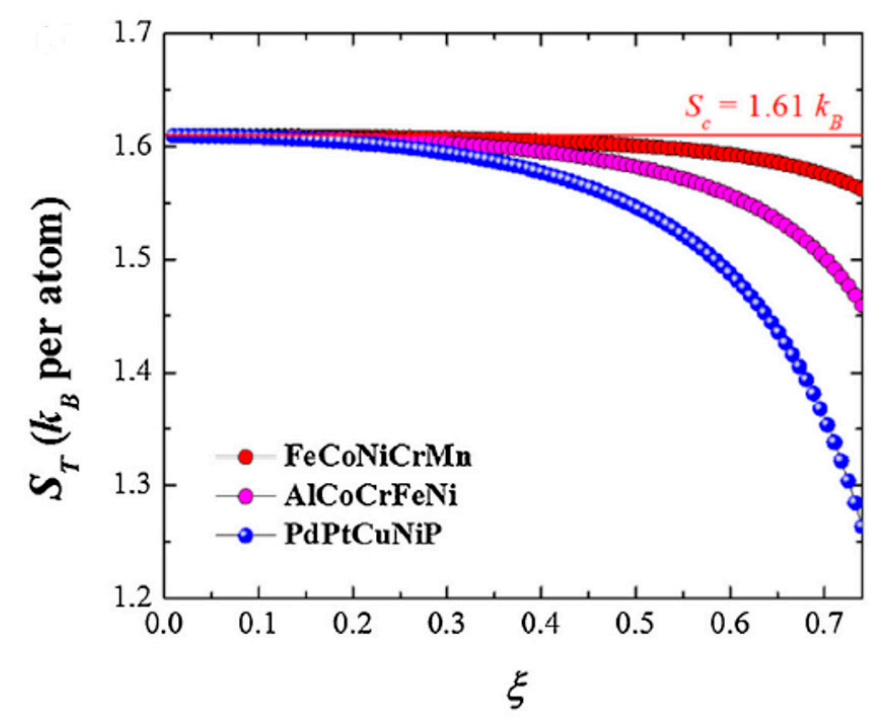

Figure 2. Variation of the total entropy of mixing, $S_{T}$, with the atomic packing fraction $\mathrm{j}$ for three fivecomponent alloys of equiatomic composition (from [9]). Reprinted with permission from Elsevier: Mater. Today. 19, Copyright 2021, License: 5047200903826.

\subsection{2. $\Omega$ Parameter}

Zhang et al. [17] proposed the $\Omega$ parameter, which combines the effects of $\Delta S_{m i x}$ and $\Delta H_{\text {mix }}$ on the stability of a multicomponent solid solution, taking the various component's melting points into consideration. The $\Omega$ and $T_{m}$ parameters are defined by

$$
\begin{aligned}
& \Omega=\frac{T_{m} \times \Delta S_{\text {mix }}}{\left|\Delta H_{\text {mix }}\right|} \\
& T_{m}=\sum_{i=1}^{N} x_{i}\left(T_{m}\right)_{i}
\end{aligned}
$$

where $T_{m}$ is the average melting temperature of the $\mathrm{N}$-element alloy and $\left(T_{m}\right)_{i}$ is the melting point of the $i$ th component of the alloy.

Similar to how Yeh et al. [1] suggested $\Delta S_{\text {mix }}>1.6 R$ as a criterion for high-entropy alloys, Zhang et al. proposed $\Omega>1.1$, where the higher the $\Omega$ value, the higher the probability of forming a single-phase random solid solution in high-entropy alloys. The parameter's potential for such estimation was originally intended to be used along with the atomic size difference parameter, $\delta$, which is described below.

\subsubsection{Atomic Size Difference}

The atomic size effect has been thoroughly corroborated and defined by several experimental data throughout the literature [17], and the critical atomic size difference $(\delta)$ found for a solid solution to form high-entropy alloys is $\delta \leq 6.6 \%$. $\delta$ is defined as [13]

$$
\delta=\sqrt{\sum_{i=1}^{N} x_{i}\left(1-\frac{d_{i}}{\sum_{j=1}^{N} x_{j} d_{j}}\right)^{2}}
$$


where $N$ is the number of elements, $x_{i}$ or $x_{j}$ is the composition of the $i$ th or $j$ th element, and $d_{i}$ or $d_{j}$ is the atomic diameter of the $i$ th or $j$ th component. In Figure 3 , we can see the discussed parameters used and paired with each other, having several phase overlaps. Some regions are identified, but ultimately, no definitive separation between them is achieved.
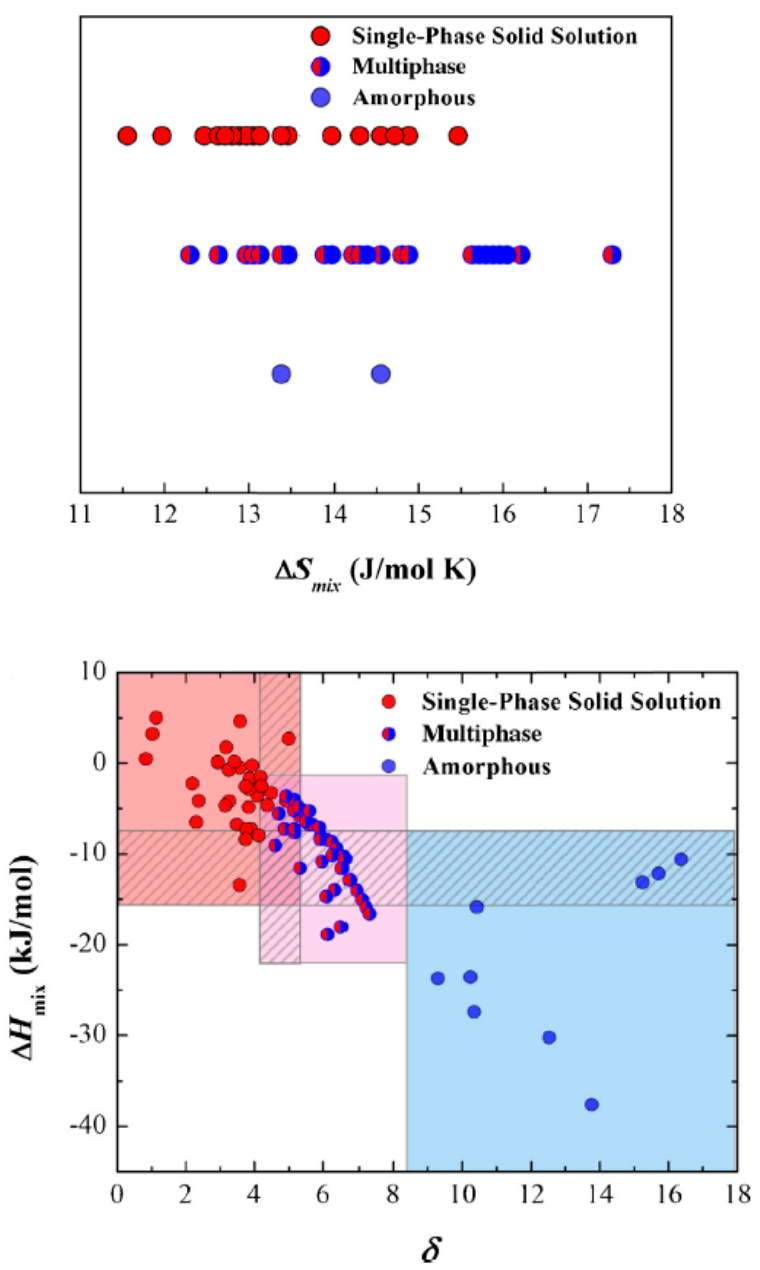
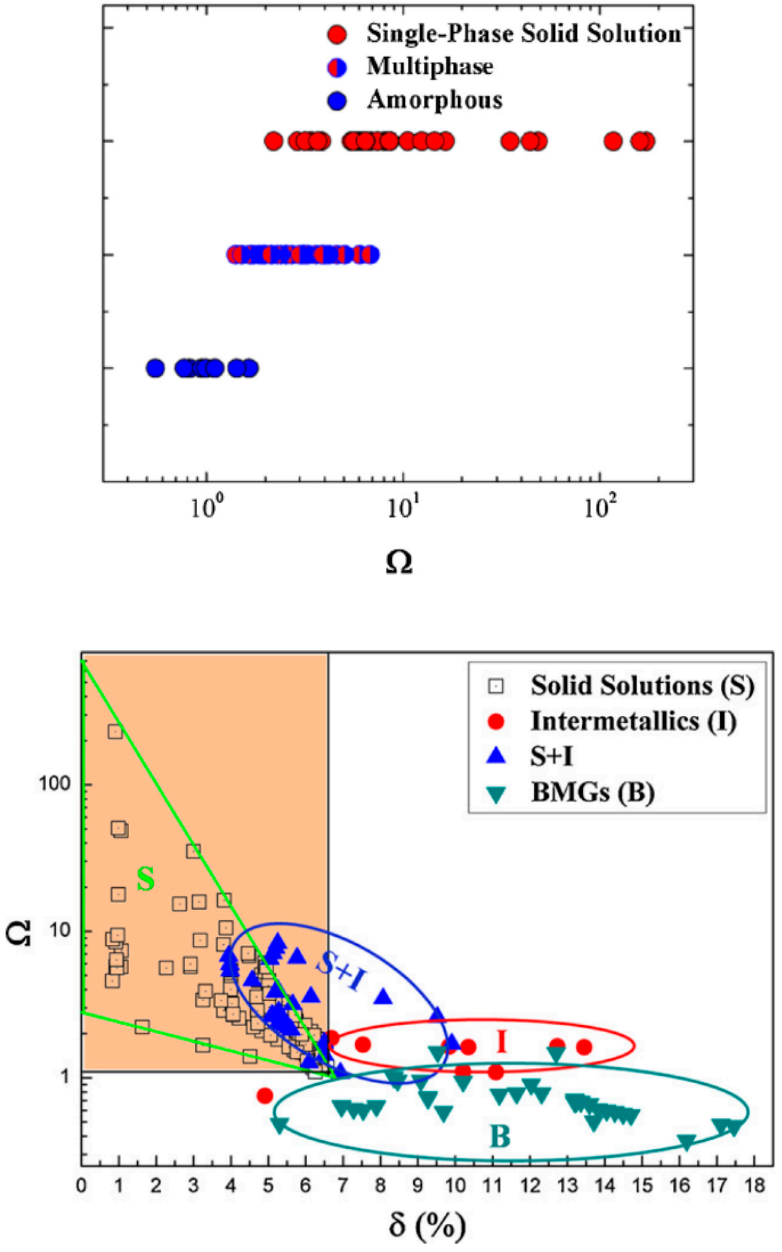

Figure 3. $\Delta S_{m i x}, \Omega$, and $\delta$ vs. $\Delta H_{m i x}$ (from [9]) and $\delta$ vs. $\Omega$ (from [17]) plots for as-cast high-entropy alloys (HEAs) with different phases, showing several phase overlaps. Reprinted with permission from Elsevier: Mater. Today. 19 (from [9]) and Mater. Chem. Phys. (from [17]), Copyright 2021, License: 5047200903826 and 5033770520802, respectively.

\subsection{4. $\Phi$ Parameter}

Ye et al. [16] pieced together the individual parameters $S_{C}$ and $S_{E}$ along with $T_{m}$ and $\Delta H_{\text {mix }}$, proposing the $\phi$ parameter, defined as

$$
\phi=\frac{S_{C}-\left|\Delta H_{m i x}\right| / T_{m}}{\left|S_{E}\right|}
$$

Both $\Delta S_{\text {mix }}$ and $\Omega$ had significant overlaps in their respective values computed for the high-entropy alloys with different phases, and $\phi$ has shown to be the best out of the three parameters, with a critical value of $\phi_{c}=20$, where alloys with higher values display single-phase solid solutions, and lower values display multiphase and amorphous solutions. Figure 4 shows the single parameter being used to successfully separate the different phase formations. 


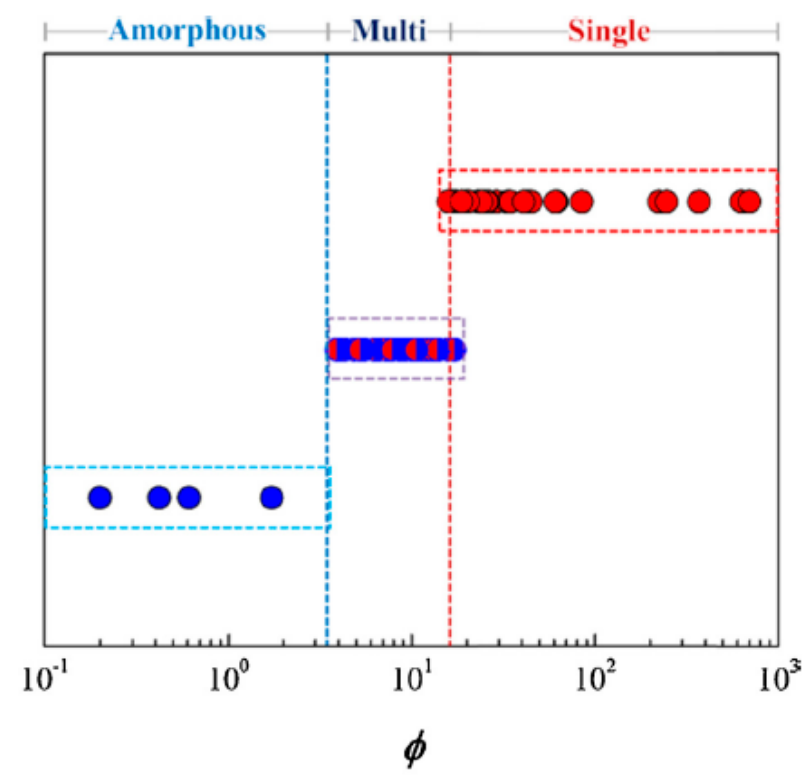

Figure 4. $\phi$ plot for different high-entropy alloy phases (from [9]). Reprinted with permission from Elsevier: Mater. Today. 19, Copyright 2021, License: 5047200903826.

\subsubsection{Valence Electron Concentration}

As described earlier, we have high entropy, causing these alloys to have mainly random solid solutions instead of intermetallic compounds, and we have a set of parameters that allows the prediction of whether they form single or multiple phases. The valence electron concentration (VEC) allows the determination of the type of structure that will be formed in high-entropy alloys, namely face-centered cubic (FCC), body-centered cubic BCC, or hexagonal close packed (HCP) structures. Figure 5 pairs both the $\phi$ parameter and the VEC, successfully separating both phase formation and crystal structure within reasonable and usable margins.

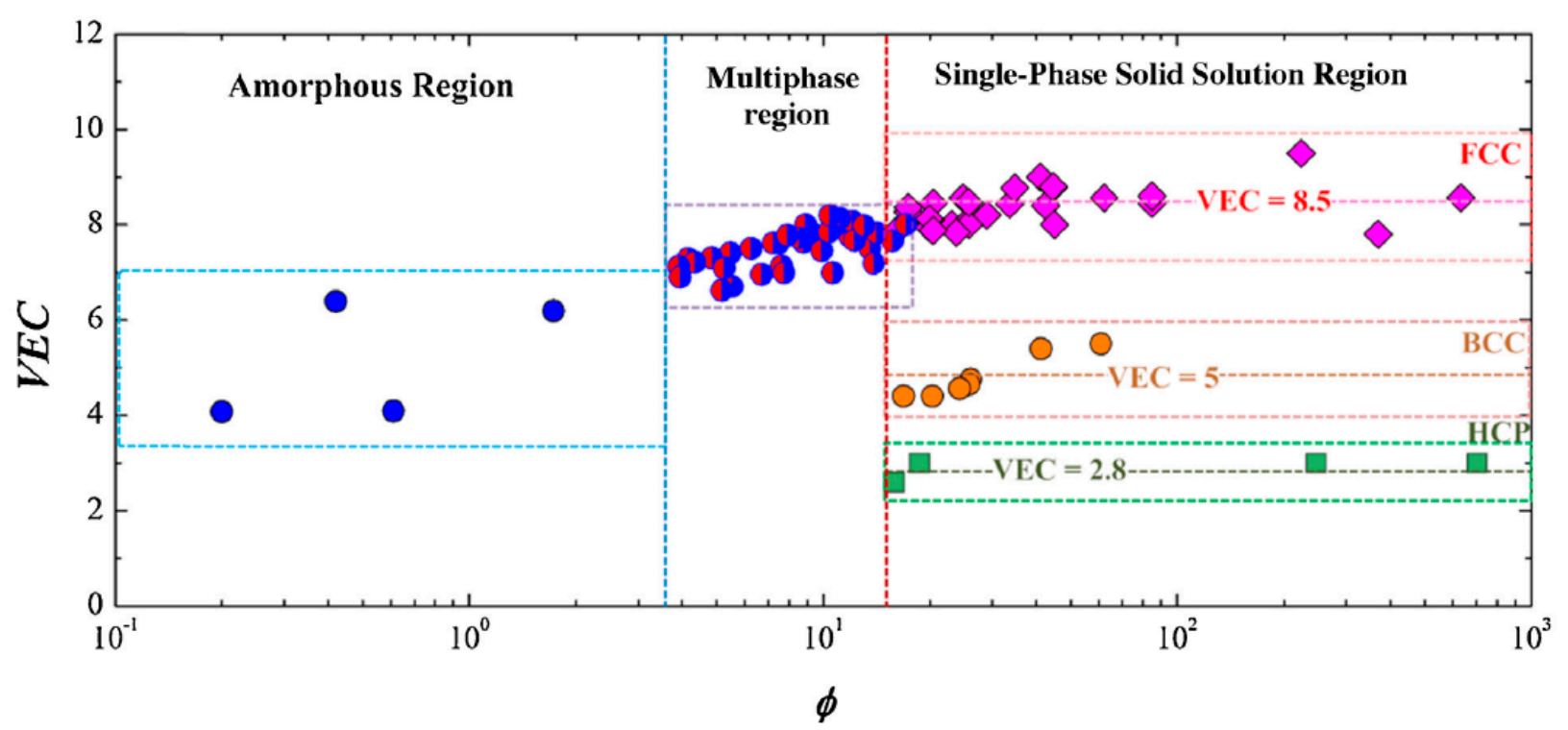

Figure 5. $\phi$ vs. valence electron concentration (VEC) plot for different high-entropy alloys (from [9]). Reprinted with permission from Elsevier: Mater. Today. 19, Copyright 2021, License: 5047200903826.

Different mean values of VEC have been found to regulate the crystal structure of existing phases in these alloys. Typically, for FCC structures, the VEC is $\approx 8.5$, while for 
BCC and HCP structures, it decreases to $\approx 5$ and $\approx 2.8$, respectively. When the d-electrons in the valence band are included, the average VEC of an alloy can be defined as:

$$
\mathrm{VEC}=\sum_{i=1}^{n} c_{i}(V E C)_{i}
$$

where $(V E C)_{i}$ is the VEC for the $i$ th element [18].

Not only is VEC a good indicator of the predominant structure for an alloy, but there are also correlations with some mechanical properties such as the ductility of conventional BCC alloys. For instance, Mo- and W-based binary alloys can be ductile if VEC decreases [18,19]. Sheikh et al. [20] reported a similar rule for high-entropy alloys, indicating a possible VEC value threshold of 4.5, where alloys with lower values would lead to high ductility, and VEC $>5$ would lead to high brittleness. These rules were studied for refractory high-entropy alloys, comprising group IV (Ti, Zr, $\mathrm{Hf}), \mathrm{V}(\mathrm{V}, \mathrm{Nb}, \mathrm{Ta})$, and $\mathrm{VI}(\mathrm{Cr}$, $\mathrm{Mo}, \mathrm{W})$ refractory elements.

\section{Biomedical Spectrum of High-Entropy Alloys}

It may come as a surprise how, after years and years of developing science and technology, we are still to find an absolute replacement for bones and joints. The mechanical properties required to withstand the constant and cyclic loads of simple daily activities such as walking without fracture are not so simple, and to top it off, the biocompatibility requirements for a material to be used as an implant are such that the possibilities are further narrowed down. Therefore, materials used in such applications must have a low modulus of elasticity, to avoid stress shielding [21]; high yield strength; high fatigue resistance; and high ductility to be able to handle loads from physical activity. Natural load bearing joints such as the knee or hip joints are extremely well lubricated, with the bone tissue having friction coefficients of 0.003-0.0015, which are very low, and last for over 70 years [22], and so the tribological properties of implants are of utmost importance. In total hip arthroplasty (THA), wear is seen as a major cause of osteolysis, leading to bone loss due to the foreign-body autoimmune response, caused by the implant's wear debris $[23,24]$. Therefore, high surface-level wear and corrosion resistance (accordingly to the contact with the tissue or body fluid) and a low coefficient of friction are also essential, besides the obvious strict and demanding biocompatibility needs [25,26]. There are a series of standards for evaluating the biocompatibility of medical devices. These standards are part of ISO 10993 [27], serving as a framework for the development of biocompatibility evaluations. Such standards are recognized worldwide, with little additional (or fewer) recommendations from regulatory agencies such as the Food and Drug Administration (FDA) and the Japanese Ministry of Labor Health and Welfare (MHLW).

The International Organization for Standardization details the necessary tests for each application, and biomedical application devices based on high-entropy alloys are mainly focused on load bearing joints, e.g., in knee and hip arthroplasty, and so the proper categorization falls into implant devices, with contact to tissue/bone with a type C duration (more than 30 days) [28]. Of course, this list does not mandate any testing whatsoever, hence the name "Endpoints to be addressed in a biological risk assessment". It simply provides the biological effects to be taken into consideration given the class of the medical device being tested. Having said this, the associated testing can be quite slow and expensive (e.g., subacute/subchronic toxicity tests) and requires careful planning and a finished device ready for market. This means that early development stages such as material design and validation do not justify long and expensive tests. There are, however, more economically and logistically reasonable tests that can be carried out in early development. In vitro tests such as cell proliferation, live/dead viability assays, osteoblast adhesion and morphology, and bio-corrosion behaviors with potentiodynamic polarization curves in Hank's or phosphate buffer solutions are common, and some studies also include in vivo tests in mice, which cover more biological effects than the in vitro ones. 
There are several alloys already used as biomedical implants. One of the most used alloys is Ti6Al4V [29], also known as Ti64, and thus, it is frequently used for comparison when alternatives are being sought. Although common, there is still room for improvement in both its mechanical and tribological properties, stress shielding, and the presence of cytotoxic elements inherent to Ti6A4IV. Such disadvantages are being tackled by the development of novel biomedical high-entropy alloys, where several mechanical and tribological properties can be improved dramatically, while retaining excellent biocompatibility.

Since 2004, there has been an exponential increase in the number of published papers on high-entropy alloys [6,8-11,30,31]. Although most of the literature pertaining to highentropy alloys is focused on structural application under a wide range of environments, it did not take long until applications for medical implants began to be considered. Next, some recent developments in this field are described and discussed.

\subsection{Comparison of Mechanical Properties for Bone Implants}

First, we have biocompatibility factors limiting the elements that are usable in the material, e.g., the requirement of high yield strength and ductility to support heavy and cyclic loads during long-term usage, corrosion, and wear resistance and the need for a low Young's modulus to avoid stress shielding, as detailed above.

Below, a list of high-entropy alloys studied for possible biomedical applications is presented (refer to Table 1). In this list, only high-entropy alloys that already had their mechanical properties determined (i.e., Young's modulus, yield strength, and ductility) are presented. Given the biocompatibility of the constituent elements in Table 1, an efficiency function (i) is presented for the mechanical properties and is obtained considering the ratio between a material's resilience modulus $U_{r}$, and $\left(E-E_{B o n e}\right)$, where $E$ is the alloy's Young modulus and $E_{\text {Bone }}$ is the corresponding elastic modulus of the bone. To simplify, $E_{\text {Bone will }}$ be regarded as the average value of $20 \mathrm{GPa}$ [25]. This is an attempt to rank and order the data for an approximation of the desired and ideal mechanical properties. Thus, we define

$$
\begin{gathered}
U_{r}=\frac{(\text { Yield strength })^{2}}{2 E} \\
i=\frac{\frac{(\text { Yield strength })^{2}}{2 E}}{\left(E-E_{\text {Bone }}\right)}
\end{gathered}
$$

It is worth noting that the simplicity of such a function and its linearity will not factor in any diminishing contributions either from yield stress or from Young modulus above or below ideal values, for the appropriate application, meaning it will not effectively measure the mechanical performance within the necessary and reasonable parameters, since those parameters differ with each biomedical application scenario.

For instance, selective laser melted $\mathrm{Ti}_{1 \cdot 4} \mathrm{Nb}_{0 \cdot 6} \mathrm{Ta}_{0 \cdot 6} \mathrm{Zr}_{1 \cdot 4} \mathrm{Mo}_{0 \cdot 6}$ [32] displays a yield strength of about $1690 \mathrm{MPa}$, which for some applications might prove to be unnecessarily high, in detriment to some other alternative with less, yet sufficient, yield strength and ductility, but with better (i.e., lower) Young modulus values to avoid stress shielding, for example, for young patients whose bone will need to regenerate at an acceptable rate for far longer. Table 1 summarizes the mechanical properties of some of the recently developed high-entropy alloys targeting biomedical applications. 
Table 1. Known materials for medical implants and some recently developed biomedical high-entropy alloys.

\begin{tabular}{|c|c|c|c|c|c|}
\hline Material & Condition & E (GPa) & $\begin{array}{l}\text { Yield Strength } \\
\text { (MPa) }\end{array}$ & Ductility (\%) & $\begin{array}{l}\text { Efficiency Function } i \\
\qquad\left(10^{-5}\right)\end{array}$ \\
\hline Ti-6Al-4V (ELI) * & Mill annealed $[25,33]$ & 110 & $850-900$ & $10-15$ & 3.87 \\
\hline Equimolar TiZrNbTaMo & As-cast arc-melted [34] & 153 & $1390 \pm 75$ & $\varepsilon_{\mathrm{p}} \approx 6$ & 4.75 \\
\hline $\mathrm{Ti}_{1 \cdot 4} \mathrm{Nb}_{0 \cdot 6} \mathrm{Ta}_{0 \cdot 6} \mathrm{Zr}_{1 \cdot 4} \mathrm{Mo}_{0 \cdot 6}$ & $\begin{array}{l}\text { Selective laser melting } \\
\text { [32] }\end{array}$ & $140 \pm 9$ & $1690 \pm 78$ & $\varepsilon_{\mathrm{t}}=1.32 \pm 0.19$ & 8.50 \\
\hline Equimolar TiZrHfNbTa & & 103 & 1200 & 12 & 8.42 \\
\hline $\begin{array}{l}\text { TiZrNbTa } \\
\text { (Alloy 1) }\end{array}$ & As-cast arc-melting in a & 89 & 970 & 23 & 7.66 \\
\hline $\mathrm{Ti}_{6 \cdot 3} \mathrm{Zr}_{6 \cdot 3} \mathrm{Nb}_{6 \cdot 3} \mathrm{Ta}$ (Alloy 2 ) & Ti-gettered high-purity & 75 & 790 & 29 & 7.56 \\
\hline $\mathrm{Ti}_{7} \mathrm{Zr}_{7} \mathrm{Nb}_{5} \mathrm{Ta}$ (Alloy 3 ) & Ar atmosphere [35]. & 69 & 780 & 26 & 9.00 \\
\hline $\mathrm{Ti}_{9} \mathrm{Zr}_{9} \mathrm{NbTa}$ (Alloy 4) & Remelted at least 6 times & 57 & 690 & 24 & 11.29 \\
\hline $\mathrm{TiZrNbTa}_{1 \cdot 62}($ Alloy 5$)$ & to ensure chemical & 93 & 1050 & 12.7 & 8.12 \\
\hline $\mathrm{TiZrNb}_{2 \cdot 33} \mathrm{Ta}_{2 \cdot 33}$ (Alloy 6 ) & homogeneity & 135 & 970 & 12.5 & 3.03 \\
\hline $\mathrm{Ta}_{0} \cdot 7 \mathrm{HfZrTi}$ & & 77 & 1046 & 14.31 & 12.46 \\
\hline $\mathrm{Ta}_{0.8} \mathrm{HfZrTi}$ & & 85 & 1120 & 4.63 & 11.35 \\
\hline$(\mathrm{TaNb})_{0 \cdot 6} \mathrm{HfZrTi}$ & & 73 & 880 & 19 & 10.01 \\
\hline
\end{tabular}

* ELI = Extra Low Interstitials, referring to grade 23 of Ti-6Al-4V.

Not all high-entropy alloys from the work done by Yuan et al. [35] are referred in Table 1. Only the ones with more promising mechanical characteristics were chosen, although it is worth noting that the high-entropy alloys with HCP or HCP + BCC structures in Yuan's work all exhibited a relatively poorer mechanical performance if we take all the three characteristics (Young modulus, yield strength, and ductility) into consideration. The values for yield strength and ductility are obtained from tensile tests in the case of Yuan et al. work. Ti6Al4V is also presented for comparison, and we can see that apart from Alloy 6 [35], all other high-entropy alloys present a better efficiency function $i$, and most of them also have a better overall performance if you factor in ductility. There is no constituent element in these HEAs that has any cytotoxicity individually.

In the TiZrNbTa system, high values of Ti and $\mathrm{Zr}$ not only led to low values of the Young's modulus but also had lower yield strength compared to alloys 1 and 5. High values of $\mathrm{Nb}$ and $\mathrm{Ta}$ (alloy 6) had a sharp increase in Young modulus with only a slight decrease in yield strength.

In the $\mathrm{Ta}_{\mathrm{x}} \mathrm{HfZrTi}$ system, a single BCC structure was observed only for $\mathrm{x}=0.7,0.8$, and 1 , and the last two had lower ductility $(<5 \%)$. As such, $\mathrm{Ta}_{0} \cdot{ }_{7} \mathrm{HfZrTi}$ was the alloy with the highest $i$ value, having a good combination of Young modulus and yield strength.

From the $(\mathrm{TaNb})_{\mathrm{x}} \mathrm{HfZrTi}$ system, where $\mathrm{x}=0,0.2,0.4,0.5,0.6,0.8$, and 1 , which was experimentally tested in Yuan et al. [35], only $x=0.6$ and $x=1$ are in the table, since all other conditions had higher Young's modulus, similar yield strengths, and lower ductility (except for $\mathrm{x}=0.2$, which had $32.5 \%$ ).

Both selective laser melted $\mathrm{Ti}_{1 \cdot 4} \mathrm{Nb}_{0 \cdot 6} \mathrm{Ta}_{0} \cdot \mathrm{Zr}_{1 \cdot 4} \mathrm{Mo}_{0 \cdot 6}$ and equimolar TiZrNbTaMo have shown a rather small ductility ( $\varepsilon_{\mathrm{t}}=1.32 \%$ and $\varepsilon_{\mathrm{p}} \approx 6 \%$, respectively) compared to the rest of the materials in Table 1 (excluding the $\mathrm{Ta}_{0.8} \mathrm{HfZrTi}$ configuration). Wang et al. [34] suggests that brittleness and limited plasticity under deformation warrants further development of this system, away from equiatomic compositions.

More developments that have been occurring in this field, but that are not included in Table 1 , are discussed below.

Gurel et al. [36] explored the TiTaHfNb, TiTaHfNbZr, and TiTaHfMoZr systems in terms of mechanical properties and fracture behavior under impact loading (Charpy Vnotch test). All three BCC-structured alloys show a relatively lower Young's modulus when compared to more conventional implants. TiTaHfNb high-entropy alloy (composition of $\mathrm{Ti}_{77 \cdot 11} \mathrm{Ta}_{6} \cdot{ }_{44} \mathrm{Hf}_{6.63} \mathrm{Nb}_{12.73}$, in at.\%) had the highest energy absorption capacity and highest ductility, out of the three systems, and under impact loading, significant difference has been observed in the dislocation activity in the microstructure when adding both $\mathrm{Zr}$ and Mo to the system. Both elements had negative impact in terms of the energy absorption and brittleness of the TiTaHfNb and TiTaHf alloy systems. With a composition 
of $\mathrm{Ti}_{77 \cdot 11} \mathrm{Ta}_{6 \cdot 54} \mathrm{Hf}_{6 \cdot 63} \mathrm{Nb}_{12 \cdot 73}$, this high-entropy alloy has a measured Young modulus of 112.2 GPa, a Charpy V-notched average impact energy of $14.8 \mathrm{~J}$, and a hardness value of 3.5 GPa (using a Berkovich type indenter).

The TiTaHfNbZr system was also studied by Yang et al. [37]. An equiatomic composition processed by usual vacuum arc melting under a Ti-gettered Ar atmosphere, was tested for its biocorrosion behavior in a simulated physiological environment and in vitro biocompatibility. It showed a passivating behavior with low passive current density of about $0.08 \times 10^{-2} \mathrm{~A} / \mathrm{m}^{2}\left(0.07 \times 10^{-2} \mathrm{~A} / \mathrm{m}^{2}\right.$ for Ti6Al4V), a corrosion rate of $5.6 \times 10^{-4} \mathrm{~mm} /$ year $\left(5.9 \times 10^{-4} \mathrm{~mm} /\right.$ year for Ti6Al4V), and high electrochemical impedance in Hank's solution at $310 \mathrm{~K}$. MTT assay results for MC3T3-E1 cell-proliferation activities on the TiZrHfNbTa HEA and Ti-6Al-4V alloy after 1, 3, 5, and 7 days of incubation show that the high-entropy alloy has a slightly better cell viability, although not statistically significant. The authors did not mention this, but as seen in this review, there are several alternatives to this system and composition that reveal better biocompatibility when compared to the commercial Ti6Al4V, not to mention the high cost of constituent elements such as Hf.

A variant of the TiTaHfNbZr high-entropy alloy, $\mathrm{Ti}_{1.5} \mathrm{ZrTa}_{0.5} \mathrm{Hf}_{0.5} \mathrm{Nb}_{0.5}$ (with the equimolar sample named RHEA1 (refractory high entropy alloy) and the non-equimolar one being RHEA2) has been experimentally tested, and its properties were compared to that of the conventional $316 \mathrm{~L}$ stainless steel, CoCrMo, and Ti6Al4V alloys, namely in terms of wear resistance, wettability, pitting, and general corrosion resistance [38]. With a continuous passive plateau up to $1800 \mathrm{mV}_{\mathrm{Ag} / \mathrm{AgCl}}$ without the pitting effect and more positive $\mathrm{E}_{\mathrm{corr}}$ and lower $\mathrm{I}_{\text {corr }}$ than that of Ti6Al4V (which starts gradually exhibiting pitting corrosion behavior at $863.0 \mathrm{mV}_{\mathrm{Ag} / \mathrm{AgCl}}$ ), this alloy shows considerably higher pitting corrosion resistance and general corrosion resistance. Compared to its equimolar counterpart, the $\mathrm{Ti}_{1.5} \mathrm{ZrTa}_{0.5} \mathrm{Hf}_{0.5} \mathrm{Nb}_{0.5}$ has a significantly thicker corrosion-resistant barrier oxide film, which is attributed to a higher content of electronegative elements such as $\mathrm{Ti}$ and $\mathrm{Zr}$ and also a lower lattice strain $(0.0037$, compared to the 0.0306 from the equimolar counterpart, which is connected to the higher amount of differently sized elements such as $\mathrm{Hf}, \mathrm{Nb}$, and Ta). The contact angles measured in the sessile drop test with phosphate buffered saline (PBS) for 316 L, CoCrMo, Ti6Al4V, RHEA1, and RHEA2 were 75.4, 73.3, 56.9, 59.5, and $61.9^{\circ}$, respectively, showing a similar wettability with $\mathrm{Ti} 6 \mathrm{Al} 4 \mathrm{~V}$, and a superior one to $316 \mathrm{~L}$ and CoCrMo. Its elastic modulus (E) and hardness (H) were 98.57 and $3.02 \mathrm{GPa}$, respectively. This hardness value was taken after a pile-up correction made using AFM 3D imaging after nanoindentation tests. This pile-up was observed to be higher in intensity on the conventional materials than on both high-entropy alloys. The wear resistance was inferred trough $\mathrm{H} / \mathrm{E}$ and $\mathrm{H}^{3} / \mathrm{E}^{2}$ ratios, shown in Table 2 and found to be better than the 316 L, CoCrMo, and Ti6Al4 materials. With a superior wear resistance, similar wettability, lower Young's modulus, and significantly better corrosion resistance, this high-entropy alloy system's configuration $\left(\mathrm{Ti}_{1} \cdot \mathrm{ZrTa}_{0} \cdot \mathrm{Hf}_{0} \cdot \mathrm{Nb}_{0} \mathrm{Nb}_{5}\right)$ has shown promising potential in the biomedical field and warrants further investigation into its biocompatibility.

Table 2. Mechanical properties of $316 \mathrm{~L}$, CoCrMo, Ti6Al4V, RHEA1 (TiZrTaHfNb equimolar sample) and RHEA2 (Ti1.5ZrTa0.5Hf0.5Nb0.5 non-equimolar sample) measured by nanoindentation after applying a pile-up correction (from [38]). Reprinted with permission from Elsevier: Intermetallics, Copyright 2021, License: 5044280969444.

\begin{tabular}{ccccc}
\hline Alloy & H (GPa) & E (GPa) & H/E & H $^{\mathbf{3} / \mathbf{E}^{2}}$ \\
\hline 316 L & $2.44 \pm 0.18$ & $195.62 \pm 9.32$ & 0.012 & 0.00038 \\
CoCrMo & $4.21 \pm 0.15$ & $222.72 \pm 6.70$ & 0.019 & 0.0015 \\
Ti6Al4V & $3.32 \pm 0.15$ & $128.20 \pm 8.14$ & 0.026 & 0.0022 \\
RHEA 1 & $3.14 \pm 0.12$ & $112.74 \pm 4.66$ & 0.028 & 0.0024 \\
RHEA 2 & $3.02 \pm 0.11$ & $98.57 \pm 4.18$ & 0.031 & 0.0028 \\
\hline
\end{tabular}

Based on the TiNbTaZr system, Todai et al. [39] designed a TiNbTaZrMo alloy from several TiNbTaZrX alternatives $(\mathrm{X}=\mathrm{Cr}, \mathrm{V}, \mathrm{Mo}, \mathrm{W}, \mathrm{Fe})$, using some parameters in the 
literature $\left(\Delta S_{\text {mix }}, \Delta H_{\text {mix }}, \delta, \Omega\right.$, and VEC). The authors found that the $\mathrm{Cr}, \mathrm{V}$, and Fe options had a large atomic size difference, from the $\delta$ parameter. Mo and $W$, however, had a high possibility for the formation of a solid solution, and Mo was eventually chosen for the fact it was selected in conventional metallic biomaterials [40-44]. The TiNbTaZrMo system was similar in its elemental segregation in the DR/IR (dendrite/inter-dendrite) zones both in its as-cast and annealed form, but both mechanical and biocompatibility tests have shown what was stated to be promising potentials for HEAs in the biomedical sphere. Next are two studies on this system, discussing possible property trends in composition shifts.

Nagase et al. [45] explored the TiNbTaZr and TiZrHfMo systems, designing and producing TiZrHfCr ${ }_{0.2} \mathrm{Mo}$ and $\mathrm{TiZ}_{\mathrm{rHfCo}} \cdot 07 \mathrm{Cr}_{0} \cdot 07 \mathrm{Mo}$ systems (arc melted), and comparing them to TiNbTaZr and TiNbTaZrX $(X=V$, Mo and $W)$ in terms of hardness, and to $316 \mathrm{~L}$, CoCrMo, and CP-Ti in terms of biocompatibility (cell density on osteoblast cultivation for $24 \mathrm{~h}$ ). Both TiZrHfCr ${ }_{0 \cdot 2} \mathrm{Mo}$ and $\mathrm{TiZrHfCo}_{0.07} \mathrm{Cr}_{0 \cdot 07} \mathrm{Mo}$ were found to have a hardness of 531 and $532 \mathrm{HV}$, respectively, while having a lower liquidus temperature, $T_{L}$, than the TiNbTaZr and TiNbTaZrX counterparts. The cell density evaluations have shown that both the produced high-entropy alloys presented a lower, yet statistically similar, level of biocompatibility.

Akmal et al. [46] studied the (MoTa) $)_{x} N b T i Z r$ (TiZrNbTaMo) system for various values of $\mathrm{x}$. A critical concentration of $\mathrm{x}=0.4$ was determined, where higher concentrations of Mo and Ta led to increasing brittleness and elastic modulus and decreasing ductility. The optimal concentration was found to be $x=0.2$. The in vivo biocompatibility tests and the soft tissue response of the alloy $(x=0.2)$ showed no signs of toxic response in the performed tests. The electrochemical behavior in PBS at room temperature showed superior corrosion resistances relative to the commercial $316 \mathrm{~L}$ stainless steel, with passivity values of $1.2 \mathrm{~V}$ without signs of pitting.

Variants of the TiZrNbTaMo system were also studied by Hua et al. [47] with a molar ratio of $0.5,1,1.5$, and 2 for the Ti concentration. These high-entropy alloys showed a dendritic structure with two BCC solid solution phases. The $0.5 \mathrm{Ti}$ molar ratio alloy presented a hardness of about $500 \mathrm{HV}$ and a compressive strength of nearly $2600 \mathrm{MPa}, \mathrm{a}$ plastic strain of over $30 \%$, and the best corrosive wear resistance among the four alloys studied. This molar ratio had a rather coarsened dendrite structure relative to the rest in this study. The increase in Ti was also linked to the formation of short and round dendrite arms, attributed to the increasing fraction of the low-melting-temperature element. Corrosion wise, the alloys tested presented similar corrosion current densities in PBS solution and better corrosion potentials, meaning higher passivation film stability. XPS results show mainly $\mathrm{Ti}^{4+}, \mathrm{Zr}^{4+}, \mathrm{Nb}^{5+}, \mathrm{Ta}^{5+}, \mathrm{Mo}^{4+}$, and $\mathrm{Mo}^{6+}$ oxides in the surface film of the HEAs. The literature indicates that high concentrations of $\mathrm{Ti}, \mathrm{Zr}$, and $\mathrm{Nb}$ can lead to passivation, protecting the bare metal from corrosion $[38,48]$, and uniform distribution of Mo across different phases is also linked to dense and stable passivation films [49].

\subsection{Coatings}

Aside from producing bulk high-entropy alloys, some researchers have also devoted their time and efforts to develop these alloys via coating-based technologies. These are discussed now.

Braic et al. [26] took on TiZrNbHfTa-based system coatings, (TiZrNbHfTa)N and (TiZrNbHfTa)C, and investigated their biocompatibility. These were, as stated, the first high-entropy alloy coatings to be considered as biomaterials. The produced films were found to be composed of simple FCC solid solutions with a (111) preferred orientation. Simulated body fluid (SBF) testing revealed the carbide coating with the highest non-metal to metal ratio (carbon/metal $\approx 1.3$ ) to have the highest hardness $(\approx 31 \mathrm{GPa}$ ), best friction behavior $(\mu=0.12)$, and the highest wear resistance. Biocompatibility tests have also shown good adherence from osteoblast cells to the coating with no cytotoxic responses and a very high ratio of live cells compared with those that were found dead for all the investigated groups. 
In 2019, Tüten et al. [50] followed a previous study that first explored radio frequency (RF) magnetron sputtering using TiTaHfNbZr equimolar targets on NiTi substrates, but did not the analyze wear and corrosion resistances [51]. Tüten studied the equimolar TiTaHfNbZr high-entropy alloy thin film deposited on a Ti6Al4V substrate by RF magnetron sputtering. The coating exhibited high hardness and elastic modulus $(12.51 \pm 0.34 \mathrm{GPa}$ and $181.3 \pm 2.4 \mathrm{GPa}$, respectively), with enhanced results in wear resistance and coefficient of friction. The small grain size and uniform amorphous structure were attributed as the main strengthening mechanisms, corroborating previous studies on the hardness enhancement in amorphous-structured films [52,53]. This study corroborated an inverse trend observed in thin films, where increased surface roughness led to a lower coefficient of friction and wear rate, suggesting that further work must be done to address this.

Guo et al. [49] used a cluster-plus-glue-atom model to successfully design a refractory high-entropy alloy, (TiZrNb) ${ }_{14} \mathrm{SnMo}$ both in bulk (via vacuum arc melting) and as a coating in a pure-Ti substrate (via laser cladding). The TiZrNb system used an average virtual element $\mathrm{M}$ as solvent, since they have weak interactions between them, and then used $\mathrm{Sn}$ and Mo as the cluster-center solute atom and glue atom, respectively, since out of the two, $\mathrm{Sn}$ has the strongest interactions with the average virtual element's constituents ( $\mathrm{Ti}, \mathrm{Zr}$, and $\mathrm{Nb}$ ). The bulk high-entropy alloy consisted of both BCC dendritic phase and rod-like HCP$(\mathrm{Sn}, \mathrm{Zr})$ ordered phase. The coating had reduced elemental segregation between dendrite (DR) and inter-dendrite (IR), as expected, and so, this ordered phase was fully restrained in the as-clad coating. The coating had a lower Young's modulus $\left(E_{D R}=97.9 \mathrm{GPa}\right.$ and $\left.E_{I R}=88.6 \mathrm{GPa}\right)$ than its bulk counterpart $\left(E_{D R}=116.65 \mathrm{GPa}\right.$ and $\left.E_{I R}=110.47 \mathrm{GPa}\right)$. Wear rate, corrosion, wettability, and cell adhesion tests were performed. Both bulk and coating outperformed Ti6Al4V on all those tests. For example, the coating's wear volume loss was less than a third of that of Ti6Al4V and with almost double the cell covering area ratio for the 7 days of cell culture for the growth morphology test of MG-63 cells, indicating an excellent candidate for a biomaterial. Both the coating and bulk refractory high entropy alloys (RHEAs) revealed high hardness.

\subsection{Elemental Homogenization}

Alternative processing routes are also being explored to enhance the properties of high-entropy alloys via elemental homogenization of the microstructure, as will be discussed now.

Perumal et al. [54] used stationary friction processing (SFP) on a MoNbTaTiZr HEA, managing to homogenize surface-level elemental distribution to a degree of efficiency not possible by annealing. In about $15 \mathrm{~min}$, they achieved a level of elemental homogenization across dendritic and inter-dendritic regions equivalent to an annealing treatment at $1275 \mathrm{~K}$ for a whole week. Biocorrosion resistance tests have shown a near sixfold increase compared to the as-cast counterpart, with a similar result for biocompatibility tests, which indicates higher viability compared to both the as-cast counterpart and the more conventional biomaterials such as $316 \mathrm{~L}$ stainless steel and Ti6Al4V.

In hopes to improve the microstructure's homogeneity, powder metallurgy (PM) routes have also been tried [55]. Popescu et al. explored the TiZrNbTaFe system with $\mathrm{PM}$ method and further corroborated the findings that additions of $\mathrm{Nb}$ and $\mathrm{Zr}$ to a Ti matrix lead to the formation of a multicomponent alloy with an extremely high resistance to active and passive dissolution in acids, as stated by Yu et al. [56]. The corrosion rates were notably better than that of Ti6Al4V, but direct comparisons to its as-cast counterpart was not experimentally made. It does, however, suggest some potential in TiZrNbTaFe systems for biomedical applications. Although some useful information was found in this work, there was no elemental analysis of the resulting microstructure to be able to compare the effect on segregation mitigation against other processing routes.

Selective laser melting has also received some attention for its high cooling rate characteristics [32,57]. Ishimoto et al. compared an as-cast and a selective laser melting built $\mathrm{Ti}_{1 \cdot 4} \mathrm{Nb}_{0 \cdot 6} \mathrm{Ta}_{0 \cdot 6} \mathrm{Zr}_{1 \cdot 4} \mathrm{Mo}_{0 \cdot 6}$ high-entropy alloy [32]. The two differed both in microstructure 
and mechanical properties. The additively manufactured alloy had a very low level of elemental segregation compared to the as-cast counterpart. The dendrite structure was compared for chemical composition and results are shown in Table 3. Compared to the ascast counterpart, the selective laser melted part also showed improved osteoblast adhesion, better compressive yield strength (1690 from $1140 \mathrm{MPa})$, and a true strain at break of $1.32 \pm 0.19 \%$, this was attributed to the very refined microstructure induced by the high cooling rate induced by selective laser melting.

Table 3. Comparison of the local chemical composition (at.\%) of a selective laser melting (SLM) and as-cast $\mathrm{Ti}_{1.4} \mathrm{Nb}_{0.6} \mathrm{Ta}_{0.6} \mathrm{Zr}_{1.4} \mathrm{Mo}_{0.6}$ biomedical high-entropy alloy (from [32]).

\begin{tabular}{ccccccc}
\hline \multicolumn{2}{c}{ In at. $\%$. } & Ti & Nb & Ta & Zr & Mo \\
\hline \multicolumn{2}{c}{ Nominal concentration } & 30.4 & 13.0 & 13.0 & 30.4 & 13.0 \\
\multirow{2}{*}{ SLM } & Dendrite & 30.7 & 12.0 & 13.0 & 30.7 & 13.7 \\
\multirow{2}{*}{ As-cast } & Inter-dendrite & 31.4 & 10.9 & 10.7 & 34.3 & 12.6 \\
& Dendrite & 26.8 & 16.6 & 22.6 & 17.3 & 16.9 \\
& Inter-dendrite & 30.8 & 11.5 & 8.66 & 38.3 & 10.6 \\
\hline
\end{tabular}

\subsection{Biocompatibility Studies}

Thus far, most the evaluation of biomedical high-entropy alloys primarily focused on its mechanical properties and phase stability. However, some recent works have studied the in vivo and in vitro properties of these novel engineering alloys.

Akmal et al. studied the in vivo biocompatibility of alloys from the Mo-Ta-Nb-TiZr system [46]. When their optimized alloy, the selection of which was made based on mechanical properties, was used as implant in mice, it was observed that no muscle damage or adverse effects due to potential toxicity problems occurred. Importantly, one of the key conclusions from Akmal et al.'s work concerns the fact that, for this alloy system, such impacts can be considered as safe as those of key biomedical materials such as NiTi shape memory alloys or Ti-6Al-4V alloys. Yang et al. evaluated the in vitro compatibility of a TiZrHfNbTa high-entropy alloy [37]. Good cell adhesion, viability, and proliferation of the MC3T3-E1 cells used in this study highlighted the potential of such an alloy to be used in biomedically oriented applications. Wang et al. studied the biocompatibility of a Ti40Zr20Hf10Nb20Ta10 high-entropy alloy for human gingival fibroblasts [58]. Overall, no cytotoxicity was demonstrated when the alloy was in contact with live tissue. Moreover, compared to its TiZrHf counterpart, the TiZrHfNbTa alloy exhibited improved proliferation of fibroblasts, which translates to better biocompatibility properties.

The lack of systematic studies on the biocompatibility of potential biomedical alloys is something that is expected to be addressed soon given the extraordinary properties of these materials. Up to now, these materials are primary candidates for biomedical applications but need further verification to establish their boundaries of application.

\section{Conclusions}

Several biomedical-oriented high-entropy alloys are now being developed, uncovering the vast pool of compositional possibilities. Existing ones still have a long way to go in their development, be it in processing method, composition, and even compatibility between components in the case of coating technologies. There are an increasing number of relevant parameters that help in the design of these materials. The entropy $\Delta S_{\text {mix }}$ and enthalpy $\Delta H_{\text {mix }}$ of mixing [1]; the atomic size difference, $\delta$ [13], $S_{T}, S_{E}$, and $S_{C}[14,15]$; the $\Phi$ parameter [16]; and VEC are still the most used, but cluster-plus-glue-atom models are also being successfully applied [49].

In this short overview, a range of mechanical properties of several biomedical highentropy alloys were compared and ranked with a proposed efficiency function, $i$. $\mathrm{Ti}_{9} \mathrm{Zr}_{9} \mathrm{NbTa}$ $(i=11.29)$, $\mathrm{Ta}_{0.8} \mathrm{HfZrTi}(i=11.35)$, and $\mathrm{Ta}_{0} \cdot{ }_{7} \mathrm{HfZrTi}(i=12.46)$ were at the top, with $\mathrm{Ta}_{0.8} \mathrm{HfZrTi}$ having a relatively lower ductility. By comparison, Ti6Al4V had an $i=3.87$. It must be noted that this list is very short, since the amount of standardized information 
regarding basic mechanical properties such as yield strength, Young's modulus, and ductility are not yet abundant in the literature, since most studies focus on other pressing issues such as corrosion behavior and biocompatibility. Therefore, finding reliably comparable data is not elementary. It is to be noted, however, that most biomedical high-entropy alloys discussed here have superior mechanical behavior than the commercially available Ti6Al4V, $316 \mathrm{~L}$ stainless steel, and CoCrMo alternatives.

Rapid cooling seems to be a standard goal for most processing methods due to the advantages in suppressing elemental segregation [10,32,49]. Rapid cooling processes such as selective laser melting are receiving some attention for that reason, resulting in grain refinement and enhanced solid solution strengthening [57]. While coating production does not have the same cooling speed as those found during selective laser melting, interesting properties are still obtained in the materials obtained by these methods.

Other approaches have been made to mitigate elemental segregation, including the powder metallurgy route by Popescu et al. [55] and stationary friction processing by Perumal et al. [54]. It is worth nothing that the latter had biocorrosion resistance tests show a near sixfold increase compared to the as-cast counterpart (MoNbTaTiZr system). Therefore, elemental homogenization is indeed a relevant part in further improving both high-entropy alloys and their associated biomedical properties.

In summary, the future of biomedical high-entropy alloys is promising, though there is a need for a more in-depth analysis of the microstructure/properties relationships for these alloys.

Author Contributions: Conceptualization: J.P.O.; Investigation: D.C., P.J., A.C.B., J.P.O.; Writingoriginal draft preparation: D.C.; Writing—review and editing: A.C.B., J.P.O. All authors have read and agreed to the published version of the manuscript.

Funding: This research was funded by FCT-MCTES, grant numbers UIDB/50025/2020-2023 and UIDB $/ 00667 / 2020$.

Acknowledgments: This work was partially funded by national funds through the FCT—Fundação para a Ciência e a Tecnologia, I.P., under the scope of the project UIDB/50025/2020-2023. J.P.O. acknowledges Fundação para a Ciência e a Tecnologia (FCT-MCTES) for its financial support via the project UIDB/00667/2020 (UNIDEMI).

Conflicts of Interest: The authors declare no conflict of interest.

\section{References}

1. Yeh, J.-W.; Chen, S.-K.; Lin, S.-J.; Gan, J.-Y.; Chin, T.-S.; Shun, T.-T.; Tsau, C.-H.; Chang, S.-Y. Nanostructured High-Entropy Alloys with Multiple Principal Elements: Novel Alloy Design Concepts and Outcomes. Adv. Eng. Mater. 2004, 6, 299-303. [CrossRef]

2. Cantor, B.; Chang, I.; Knight, P.; Vincent, A. Microstructural development in equiatomic multicomponent alloys. Mater. Sci. Eng. A 2004, 375-377, 213-218. [CrossRef]

3. Otto, F.; Yang, Y.; Bei, H.; George, E. Relative effects of enthalpy and entropy on the phase stability of equiatomic high-entropy alloys. Acta Mater. 2013, 61, 2628-2638. [CrossRef]

4. Gali, A.; George, E. Tensile properties of high- and medium-entropy alloys. Intermet. 2013, 39, 74-78. [CrossRef]

5. Miracle, D.B.; Miller, J.D.; Senkov, O.N.; Woodward, C.; Uchic, M.D.; Tiley, J. Exploration and Development of High Entropy Alloys for Structural Applications. Entropy 2014, 16, 494-525. [CrossRef]

6. High-Entropy Alloys; Springer Science and Business Media LLC: New York, NY, USA, 2016.

7. Ranganathan, S. Alloyed pleasures: Multimetallic cocktails. Curr. Sci. 2003, 85, 1404-1406.

8. Yeh, J.-W. Recent progress in high-entropy alloys. Eur. J. Control. 2006, 31, 633-648. [CrossRef]

9. Ye, Y.; Wang, Q.; Lu, J.; Liu, C.; Yang, Y. High-entropy alloy: Challenges and prospects. Mater. Today 2016, 19, 349-362. [CrossRef]

10. Yeh, J.-W. Alloy Design Strategies and Future Trends in High-Entropy Alloys. JOM 2013, 65, 1759-1771. [CrossRef]

11. Tsai, M.-H.; Yeh, J.-W. High-Entropy Alloys: A Critical Review. Mater. Res. Lett. 2014, 2, 107-123. [CrossRef]

12. Tong, C.-J.; Chen, Y.-L.; Yeh, J.-W.; Lin, S.-J.; Chen, S.-K.; Shun, T.-T.; Tsau, C.-H.; Chang, S.-Y. Microstructure characterization of $\mathrm{Al} \times \mathrm{CoCrCuFeNi}$ high-entropy alloy system with multiprincipal elements. Met. Mater. Trans. A 2005, 36, 881-893. [CrossRef]

13. Zhang, Y.; Zhou, Y.J.; Lin, J.P.; Chen, G.L.; Liaw, P.K. Solid-Solution Phase Formation Rules for Multi-component Alloys. Adv. Eng. Mater. 2008, 10, 534-538. [CrossRef]

14. Mansoori, G.A.; Carnahan, N.F.; E Starling, K.; Leland, T.W. Equilibrium Thermodynamic Properties of the Mixture of Hard Spheres. J. Chem. Phys. 1971, 54, 1523-1525. [CrossRef] 
15. Ye, Y.; Wang, Q.; Lu, J.; Liu, C.; Yang, Y. The generalized thermodynamic rule for phase selection in multicomponent alloys. Intermet. 2015, 59, 75-80. [CrossRef]

16. Ye, Y.; Wang, Q.; Lu, J.; Liu, C.; Yang, Y. Design of high entropy alloys: A single-parameter thermodynamic rule. Scr. Mater. 2015, 104, 53-55. [CrossRef]

17. Yang, X.; Zhang, Y. Prediction of high-entropy stabilized solid-solution in multi-component alloys. Mater. Chem. Phys. 2012, 132, 233-238. [CrossRef]

18. Guo, S.; $\mathrm{Ng}, \mathrm{C} . ; \mathrm{Lu}, \mathrm{J} . ; \mathrm{Liu}, \mathrm{C} . \mathrm{T}$. Effect of valence electron concentration on stability of fcc or bcc phase in high entropy alloys. J. Appl. Phys. 2011, 109, 103505. [CrossRef]

19. Qi, L.; Chrzan, D.C. Tuning Ideal Tensile Strengths and Intrinsic Ductility of bcc Refractory Alloys. Phys. Rev. Lett. 2014, 112, 115503. [CrossRef] [PubMed]

20. Sheikh, S.; Shafeie, S.; Hu, Q.; Ahlström, J.; Persson, C.; Veselý, J.; Zýka, J.; Klement, U.; Guo, S. Alloy design for intrinsically ductile refractory high-entropy alloys. J. Appl. Phys. 2016, 120, 164902. [CrossRef]

21. Song, Y.; Xu, D.; Yang, R.; Li, D.; Wu, W.; Guo, Z. Theoretical study of the effects of alloying elements on the strength and modulus of $\beta$-type bio-titanium alloys. Mater. Sci. Eng. A 1999, 260, 269-274. [CrossRef]

22. Sreekanth, P.S.R.; Kanagaraj, S. Wear of Biomedical Implants. In Tribology for Scientists and Engineers; Metzler, J.B., Ed.; Springer: New York, NY, USA, 2013; pp. 657-674.

23. Zhu, Y.H.; Chiu, K.Y.; Tang, W.M. Review Article: Polyethylene wear and osteolysis in total hip arthroplasty. J. Orthop. Surg. 2001, 9, 91-99. [CrossRef]

24. Amstutz, H.C.; Campbell, P.; Kossovsky, N.; Clarke, I.C. Mechanism and clinical significance of wear debris-induced osteolysis. Clin. Orthop. Relat. Res. 1992, 276, 7-18. [CrossRef]

25. Long, M.; Rack, H. Titanium alloys in total joint replacement-a materials science perspective. Biomaterials 1998, 19, 1621-1639. [CrossRef]

26. Braic, V.; Balaceanu, M.; Braic, M.; Vladescu, A.; Panseri, S.; Russo, A. Characterization of multi-principal-element (TiZrNbHfTa)N and (TiZrNbHfTa)C coatings for biomedical applications. J. Mech. Behav. Biomed. Mater. 2012, 10, 197-205. [CrossRef]

27. CSA. New 10993-1 Evaluation and testing within a risk management process. Int. Organ. 2007, 1-11.

28. International Organization for Standardization. ISO 10993-1:2018-Biological Evaluation of Medical Devices Part 1: Evaluation and Testing within a Risk Management Process. 2018. Available online: https:/ / www.iso.org/standard/68936.html (accessed on 30 March 2021).

29. Oliveira, J.; Panton, B.; Zeng, Z.; Andrei, C.; Zhou, Y.; Miranda, R.; Fernandes, F.B. Laser joining of NiTi to Ti6Al4V using a Niobium interlayer. Acta Mater. 2016, 105, 9-15. [CrossRef]

30. Zhang, Y.; Zuo, T.T.; Tang, Z.; Gao, M.C.; Dahmen, K.A.; Liaw, P.K.; Lu, Z.P. Microstructures and properties of high-entropy alloys. Prog. Mater. Sci. 2014, 61, 1-93. [CrossRef]

31. Derimow, N.A.; Abbaschian, R. Liquid Phase Separation in High-Entropy Alloys-A Review. Entropy 2018, 20, 890. [CrossRef]

32. Ishimoto, T.; Ozasa, R.; Nakano, K.; Weinmann, M.; Schnitter, C.; Stenzel, M.; Matsugaki, A.; Nagase, T.; Matsuzaka, T.; Todai, M.; et al. Development of TiNbTaZrMo bio-high entropy alloy (BioHEA) super-solid solution by selective laser melting, and its improved mechanical property and biocompatibility. Scr. Mater. 2021, 194, 113658. [CrossRef]

33. Wang, K. The use of titanium for medical applications in the USA. Mater. Sci. Eng. A 1996, 213, 134-137. [CrossRef]

34. Wang, S.-P.; Xu, J. TiZrNbTaMo high-entropy alloy designed for orthopedic implants: As-cast microstructure and mechanical properties. Mater. Sci. Eng. C 2017, 73, 80-89. [CrossRef] [PubMed]

35. Yuan, Y.; Wu, Y.; Yang, Z.; Liang, X.; Lei, Z.; Huang, H.; Wang, H.; Liu, X.; An, K.; Wu, W.; et al. Formation, structure and properties of biocompatible TiZrHfNbTa high-entropy alloys. Mater. Res. Lett. 2019, 7, 225-231. [CrossRef]

36. Gurel, S.; Yagci, M.; Canadinc, D.; Gerstein, G.; Bal, B.; Maier, H. Fracture behavior of novel biomedical Ti-based high entropy alloys under impact loading. Mater. Sci. Eng. A 2021, 803, 140456. [CrossRef]

37. Yang, W.; Liu, Y.; Pang, S.; Liaw, P.K.; Zhang, T. Bio-corrosion behavior and in vitro biocompatibility of equimolar TiZrHfNbTa high-entropy alloy. Intermetallics 2020, 124, 106845. [CrossRef]

38. Motallebzadeh, A.; Peighambardoust, N.S.; Sheikh, S.; Murakami, H.; Guo, S.; Canadinc, D. Microstructural, mechanical and electrochemical characterization of TiZrTaHfNb and Ti1.5ZrTa0.5Hf0.5Nb0.5 refractory high-entropy alloys for biomedical applications. Intermetallics 2019, 113, 106572. [CrossRef]

39. Todai, M.; Nagase, T.; Hori, T.; Matsugaki, A.; Sekita, A.; Nakano, T. Novel TiNbTaZrMo high-entropy alloys for metallic biomaterials. Scr. Mater. 2017, 129, 65-68. [CrossRef]

40. Okazaki, Y.; Ito, Y.; Kyo, K.; Tateishi, T. Corrosion resistance and corrosion fatigue strength of new titanium alloys for medical implants without V and Al. Mater. Sci. Eng. A 1996, 213, 138-147. [CrossRef]

41. Lee, S.-H.; Hagihara, K.; Nakano, T. Microstructural and Orientation Dependence of the Plastic Deformation Behavior in $\beta$-type Ti-15Mo-5Zr-3Al Alloy Single Crystals. Met. Mater. Trans. A 2011, 43, 1588-1597. [CrossRef]

42. Lee, S.-H.; Todai, M.; Tane, M.; Hagihara, K.; Nakajima, H.; Nakano, T. Biocompatible low Young's modulus achieved by strong crystallographic elastic anisotropy in Ti-15Mo-5Zr-3Al alloy single crystal. J. Mech. Behav. Biomed. Mater. 2012, 14, 48-54. [CrossRef]

43. Chiba, A.; Kumagai, K.; Nomura, N.; Miyakawa, S. Pin-on-disk wear behavior in a like-on-like configuration in a biological environment of high carbon cast and low carbon forged Co-29Cr-6Mo alloys. Acta Mater. 2007, 55, 1309-1318. [CrossRef] 
44. Hiromoto, S.; Onodera, E.; Chiba, A.; Asami, K.; Hanawa, T. Microstructure and corrosion behaviour in biological environments of the new forged low-Ni Co?Cr?Mo alloys. Biomaterials 2005, 26, 4912-4923. [CrossRef]

45. Nagase, T.; Iijima, Y.; Matsugaki, A.; Ameyama, K.; Nakano, T. Design and fabrication of Ti-Zr-Hf-Cr-Mo and Ti-Zr-Hf-Co-Cr-Mo high-entropy alloys as metallic biomaterials. Mater. Sci. Eng. C 2020, 107, 110322. [CrossRef] [PubMed]

46. Akmal, M.; Hussain, A.; Afzal, M.; Lee, Y.I.; Ryu, H.J. Systematic Study of (MoTa)xNbTiZr Medium- and High-Entropy Alloys for Biomedical Implants- In Vivo Biocompatibility Examinations. J. Mater. Sci. Technol. 2020, 78, 183-191. [CrossRef]

47. Hua, N.; Wang, W.; Wang, Q.; Ye, Y.; Lin, S.; Zhang, L.; Guo, Q.; Brechtl, J.; Liaw, P.K. Mechanical, corrosion, and wear properties of biomedical Ti-Zr-Nb-Ta-Mo high entropy alloys. J. Alloy. Compd. 2021, 861, 157997. [CrossRef]

48. Zhou, Q.; Sheikh, S.; Ou, P.; Chen, D.; Hu, Q.; Guo, S. Corrosion behavior of Hf0.5Nb0.5Ta0.5Ti1.5Zr refractory high-entropy in aqueous chloride solutions. Electrochem. Commun. 2019, 98, 63-68. [CrossRef]

49. Guo, Y.; Li, X.; Liu, Q. A novel biomedical high-entropy alloy and its laser-clad coating designed by a cluster-plus-glue-atom model. Mater. Des. 2020, 196, 109085. [CrossRef]

50. Tüten, N.; Canadinc, D.; Motallebzadeh, A.; Bal, B. Microstructure and tribological properties of TiTaHfNbZr high entropy alloy coatings deposited on Ti 6Al 4V substrates. Intermetallics 2019, 105, 99-106. [CrossRef]

51. Motallebzadeh, A.; Yagci, M.B.; Bedir, E.; Aksoy, C.B.; Canadinc, D. Mechanical Properties of TiTaHfNbZr High-Entropy Alloy Coatings Deposited on NiTi Shape Memory Alloy Substrates. Met. Mater. Trans. A 2018, 49, 1992-1997. [CrossRef]

52. Shu, F.; Wu, L.; Zhao, H.; Sui, S.; Zhou, L.; Zhang, J.; He, W.; He, P.; Xu, B. Microstructure and high-temperature wear mechanism of laser cladded CoCrBFeNiSi high-entropy alloy amorphous coating. Mater. Lett. 2018, 211, 235-238. [CrossRef]

53. Greer, A.L.; Rutherford, K.L.; Hutchings, I.M. Wear resistance of amorphous alloys and related materials. Int. Mater. Rev. 2002, 47, 87-112. [CrossRef]

54. Perumal, G.; Grewal, H.S.; Pole, M.; Reddy, L.V.K.; Mukherjee, S.; Singh, H.; Manivasagam, G.; Arora, H.S. Enhanced Biocorrosion Resistance and Cellular Response of a Dual-Phase High Entropy Alloy through Reduced Elemental Heterogeneity. ACS Appl. Bio Mater. 2020, 3, 1233-1244. [CrossRef]

55. Popescu, G.; Ghiban, B.; A Popescu, C.; Rosu, L.; Truscă, R.; Carcea, I.; Soare, V.; Dumitrescu, D.; Constantin, I.; Olaru, M.T.; et al. New TiZrNbTaFe high entropy alloy used for medical applications. IOP Conf. Ser. Mater. Sci. Eng. 2018, 400, 022049. [CrossRef]

56. Yu, S.Y.; Scully, J.R.; Vitus, C.M. Influence of Niobium and Zirconium Alloying Additions on the Anodic Dissolution Behavior of Activated Titanium in $\mathrm{HCl}$ Solutions. J. Electrochem. Soc. 2001, 148, B68-B78. [CrossRef]

57. Mohammed, M.T.; Semelov, V.G.; Sotov, A.V. SLM-built titanium materials: Great potential of developing microstructure and properties for biomedical applications: A review. Mater. Res. Express 2019, 6, 122006. [CrossRef]

58. Wang, S.; Wu, D.; She, H.; Wu, M.; Shu, D.; Dong, A.; Lai, H.; Sun, B. Design of high-ductile medium entropy alloys for dental implants. Mater. Sci. Eng. C 2020, 113, 110959. [CrossRef] [PubMed] 